\title{
New Method for Increase of Noise Immunity of GPS Navigation Systems
}

\author{
Abdulov R.N., Asadov H.H.
}

\begin{abstract}
The paper is devoted to the developed new method for decrease of effect of specially formed jamming signals on functioning of GPS navigation system. The effect of hindering signal of jamming type transmitted by technical means of adversary side is considered. Generation of jamming signal of relevant frequency and sufficient power by adversary is carried out by the aim to reach the condition of non-possibility of receipt of GPS signals. The suggested method is based on basic equation of antenna theory, that is Frees equation. According to Frees equation the power of signal receipt by antenna of moving object with defined gain coefficient can be calculated upon transmission of signal from target objects antenna with known gain coefficient. The new parameter calculated by integration of Frees formula on passed distance supposing static character of jamming signal source position is suggested. On the basis of research of suggested parameter the target functional is formed taking into account the motionless position of jamming signal generator and non-changeable motion direction of moving object toward target containing jamming signal generator. The possible strategies for changing the jamming signal power on transition of object on route are also taken into account. It is shown that suggested parameter is of extremum feature upon proportianal or non proportional variation of gain coefficients of antennas of object and jamming signal generator: upon proportional variation the suggested parameter reaches the minimum and upon inverse identity maximum. Taking into account the extremum feature of suggested parameter the method and algorithm for decrease of effect of jamming signal on functioning of GPS navigation systems of similar technical constructions are developed.
\end{abstract}

Index Terms - Friis equation, antenna, atmosphere, error, navigation system, optimization, functional.

\section{INTRODUCTION}

As it was noted in[1], one of fundamental equations of theory of communication using antennas is Friis transmission equation. The Friis transmission equation make it possible to calculate the power received by first antenna with gain coefficient $\mathrm{G}_{1}$ upon transmission of signal from the second antenna with gain coefficient of $\mathrm{G}_{2}$. In this case the distance between antennas equals to $\mathrm{R}$, and transmission is carried out at wavelength $\lambda$ (fig.1).

Abdulov R.N., candidate of technical sciences, deputy chief engineer of Research institute of Ministry of Defense Industry of Azerbaijan Republic

Asadov H.H., doctor of technical sciences, professor, head of department of Research institute of Aerospace Informatics of National Aerospace Agency of Azerbaijan Republic

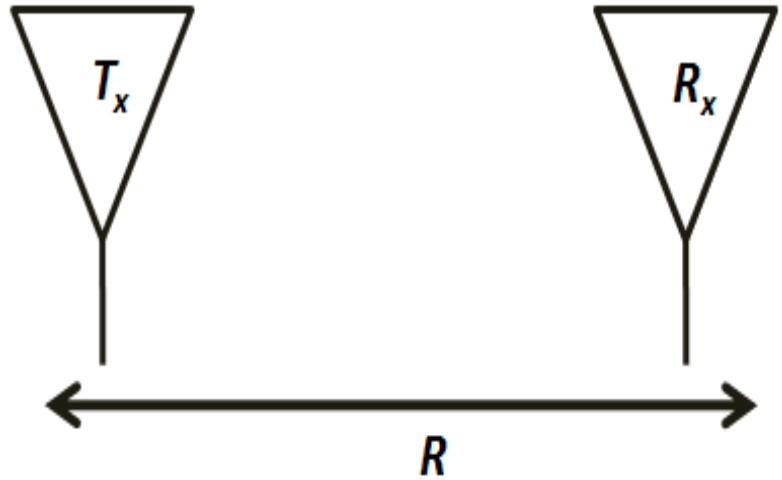

Fig.1.Transmitting $T_{x}$ and receiving $R_{x}$ antennas, located at the distance $\mathrm{R}$ between them.

According to Friis law the power of received signal could be determined as

$$
P_{R}=\frac{P_{T} \cdot G_{T} \cdot G_{R} \cdot \lambda^{2}}{\left(4 \pi R^{2}\right)}
$$

where: $\mathrm{P}_{\mathrm{T}}$-power of transmitted signal;

$\mathrm{P}_{\mathrm{R}}-$ power of received signal;

$\mathrm{G}_{\mathrm{T}}$ - gain coefficient of transmitting antenna;

$\mathrm{G}_{\mathrm{R}}$ - gain coefficient of receiving antenna.

It should be noted that formula (1) is simplified one i.e. the effect of atmospheric attenuation is not taken into account here. More strict formula for calculation of $\mathrm{P}_{\mathrm{R}}$ taking into account the atmospheric attenuation is following [2-4]:

$$
\begin{aligned}
& P_{R}=P_{T}+G_{T}\left(\theta_{T}, \varphi_{T}\right)+G_{r}\left(\theta_{r}, \varphi_{r}\right)+ \\
& +\left(\frac{\lambda}{4 \pi R}\right)^{2} \cdot\left(1-\left|\Gamma_{T}\right|^{2}\right) \cdot\left(1-\left|\Gamma_{R}\right|^{2}\right) \cdot\left|a_{T} \cdot a_{R}\right|^{2} \cdot e^{-\alpha R}
\end{aligned}
$$

where: $\left(\theta_{\mathrm{T}}, \varphi_{\mathrm{T}}\right)$-irradiation direction of transmitting antenna;

$\left(\theta_{R}, \varphi_{R},\right)$-receipt direction of receiving antenna;

$\Gamma_{\mathrm{T}}$-reflection coefficient of transmitting antenna;

$\mathrm{F}_{\mathrm{R}}$-reflection coefficient of receiving antenna;

$a_{\mathrm{T}}, \mathrm{a}_{\mathrm{R}}$ - polarization vectors of transmitting and receiving antennas, accordingly;

$\alpha-$ coefficient of attenuation of atmosphere.

According to [2], various technical complexes and systems based on GPS navigation technique are potentially subject on effect of different electromagnetic type attacks. Two types of 
attacking signals are differentiated: hindering jamming and false control spoofing signals. In first case the aim is to do impossible receiving of GPS signals by generation of signal of same frequency and sufficient power. In the second case aim is forming false control signal not differing from real signal. For example, in 2001 the technical services of Iran carry out the forced controlled landing of droneRQ-170 Sentinel, forming relevant control signal[2].

As it is noted in [5-6], GPS navigation system is based on receipt of signals from height $20000 \mathrm{\kappa m}$, which lead to low power of received signals. Due to this reason GPS receivers are subjected the effect of different type noises. If signal/noise ratio surpasses $25 \mathrm{~dB}$, GPS is not capable to

$$
P_{R}=\frac{A \cdot G_{T} \cdot G_{R}}{R^{2}}
$$

where

$$
A=\frac{P_{T} \cdot \lambda^{2}}{16 \pi^{2}}=C_{1}=\text { const }
$$

receive $(\mathrm{C} / \mathrm{A})$ code, and if said ratio equals to $55 \mathrm{~dB}$ the navigation accuracy code $(\mathrm{P})$ is loosed.[7-8]. In order to increase the noise immunity of navigation system such methods as multi-frequency systems (GPS, GALILEO, GLONAS); filtration at a single frequency; method of adaptive antennas by using of linear set antennas with maximum gain in direction to satellites and minimum gain in direction of noise generator [5, 9-10]

Next in this article we explain the theoretical basics of suggested new method for increasing the noise immunity of GPS navigation systems based on utilization of adaptively controlled antenna. The Friis formula (1) may be written as

Next we consider the situation when dependence $G_{T}=f(R)$ exists, also the distance $R$ between receiver and noise generator is variable parameter. Let us to consider following suggested integrated parameter

$$
\chi_{1}=\int_{0}^{R_{M}} P_{R} d R=\int_{0}^{R_{M}} \frac{A \cdot G_{T} \cdot G_{R}}{R^{2}} d R
$$

where: $\mathrm{R}_{\mathrm{M}}$-maximum length of distance.

Physical meaning of parameter $\chi$ is following. Assume that there is a moving object equipped with GPS/INS navigation system and static noise generator, installed at target which should be reached by moving object (it is may be guided aviation

bomb). In equation (5) $R$ is distance determined as $R=R_{0}-R_{x}$; where $R_{0}$-initial distance between the target (i.e. noise generator) and moving object; $\mathrm{R}_{\mathrm{x}}$ - current distance between them.

It is determined that the parameter $G_{R}$ should be controlled on $R$, while the law of control of $G_{T}$ is not known, but can be revealed and calculated during flight of object toward target. Two variants of adaptive variation of $G_{R}$ depending on $G_{T}$ are considered:

$$
\begin{aligned}
\text { 1. } \mathrm{G}_{\mathrm{R}} & =\mathrm{G}_{\mathrm{T}} \\
\text { 2. } \mathrm{G}_{\mathrm{R}} & =\mathrm{G}_{1}-\mathrm{G}_{\mathrm{T}}, \mathrm{G}_{1}=\text { const. }
\end{aligned}
$$

Next we assume that the aim of noise generator is synthesis of utmost hindering effective noise signals within limits of energy recourses of noise generator. As noise generation is carried out at all interval of variation of $R$, beginning from zero up to $R_{M}$ within limits of said energy resources for variation of antennas gain coefficient, the second integrated parameter could be suggested

$$
\chi_{1}=\int_{0}^{R_{M}} G_{T}(R) d R=C_{2}
$$

Where $\mathrm{C}_{2}=$ const.

Taking into account equations (5) и (8) ше can compose following equation of non-conditional variation optimization

$$
\chi_{1}=\chi_{1}+\lambda \cdot \chi_{2}=\int_{0}^{R_{M}} \frac{A \cdot G_{T}(R) \cdot G_{R}(R)}{R^{2}} d R+\lambda\left[\int_{0}^{R_{M}} G_{T}(R) d R-C_{2}\right]
$$

We should select one of equations (6) and (7), which upon some $\chi_{3}$ would reach the minimum. According to Euler-Lagrange equation the optimum function $G_{R}(R)$ should meet following condition 


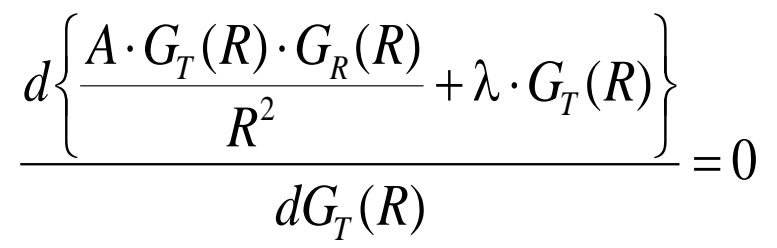

From condition (10) taking into account (6) we get

$$
\frac{2 A G_{T}(R)}{R^{2}}+\lambda=0
$$

From (11) it is derived that

$$
G_{T}(R)=\frac{-\lambda \cdot R^{2}}{-2 A}
$$

Taking into account formulas (8) and (12) we get

$$
\chi_{2}=-\int_{0}^{R_{m}} \frac{\lambda \cdot R^{2}}{2 A} d R=\frac{-\lambda \cdot R_{m}^{3}}{6 A}=C_{2}
$$

From equation(13) we have

$$
\lambda=-\frac{6 A C_{2}}{R_{m}^{3}}
$$

Taking into account formulas (11) и (14) we found

$$
\frac{2 A \cdot G_{T}(R)}{R^{2}}=\frac{6 A \cdot C_{2}}{R_{M}^{3}}
$$

Finally, from (15) we get

$$
G_{T}(R)=\frac{3 C_{2} \cdot R^{2}}{R_{m}^{3}}
$$

Thus, upon condition (16) the target functional (9) reaches its maximum. In order to check up the type of extremum it is sufficient to calculate the second derivative of integrant in (9) on $G_{T}(R)$ and to be assured that it reaches a positive that is extremum is of maximum type.

Now we study the extremum feature of functional (9) taking into account condition (7). In this case the functional (9) could be written as:

$$
\chi_{3}=\int_{0}^{R_{m}} \frac{A \cdot G_{T}(R) \cdot\left[C_{1}-G_{T}(R)\right]}{R^{2}} d R+\lambda\left[\int_{0}^{R_{m}} G_{T}(R) d R-C_{2}\right]_{(17)}
$$

According to Euler-Lagrange equation the functional (17) reaches its extremum upon function $\mathrm{G}_{\mathrm{T}}(\mathrm{R})$, which meet following condition

$$
\frac{d\left\{\frac{A \cdot G_{T}(R) \cdot\left[C_{1}-G_{T}(R)\right]}{R^{2}}+\lambda \cdot G_{T}(R)\right\}}{d G_{T}(R)}=0
$$

From condition we get (18) 


$$
\frac{A C_{1}-2 A G_{T}(R)}{R^{2}}+\lambda=0
$$

From equation (19) we get:

$$
G_{T}(R)=\frac{\lambda \cdot R^{2}+A C_{1}}{2 A}
$$

Taking into account formulas (8) и (20) wе found

$$
\int_{0}^{R_{m}}\left[\frac{\lambda R^{2}+A C_{1}}{2 A}\right] d R=C_{2}
$$

From equation (21) we find that

$$
\lambda=\frac{\left(C_{2}-\frac{C_{1}}{C_{2}} \cdot R_{m}\right) \cdot 6 A}{R_{m}^{3}}
$$

Taking into account equations (19) и (22) we get

$$
\frac{A C_{1}-2 A \cdot G_{T}(R)}{R^{2}}=\frac{\left(\frac{C_{1} \cdot R_{m}}{2}-C_{2}\right) \cdot 6 A}{R_{m}^{3}}
$$

From equation(23) it may be found that

$$
G_{T}(R)=\frac{C_{1}}{2}-\frac{3 R^{2}}{R_{m}^{3}} \cdot\left(\frac{C_{1} R_{m}}{2}-C_{2}\right)
$$

Thus upon condition (24) the target functional (17) reaches its extremum. As it was noted above, in order to determine the type of extremun the second derivative of integrant in (17) should be calculated and its sign should be determined. The negative sign of said derivative would prove that the target functional reaches its maximum.

Taking into account aforesaid following method for increasing the noise immunity of GPS navigation system is suggested. According to suggested method the system equipped with GPS navigation system should be provided with two antennas, with coefficients of gain

$$
\begin{gathered}
G_{R_{1}}(R)=G_{R}(R) \\
G_{R_{2}}(R)=C_{1}-G_{R}(R)
\end{gathered}
$$

In this case the antenna with gain coefficient $\mathrm{G}_{\mathrm{R} 2}$ is designated for joint work with noise generators antenna. The aim of such joint work is adaptive change of $\mathrm{G}_{\mathrm{R} 2}(\mathrm{R})$ in such manner that to reach the maximum of target functional

$$
\chi_{4}=\int_{0}^{R_{m}} \frac{P_{T} \cdot G_{R_{2}}(R) \cdot G_{T}(R) \cdot \lambda^{2}}{(a \pi R)^{2}} d R+\left[\int_{0}^{R_{m}} G_{T}(R) d R-C_{2}\right]
$$

Maximum of functional (27) is reached by adaptive variation of $\mathrm{G}_{\mathrm{R} 2}(\mathrm{R})$, studying dynamics of $\mathrm{G}_{\mathrm{T}}(\mathrm{R})$ and providing for inverse dynamics of variation of $\mathrm{G}_{\mathrm{R} 2}(\mathrm{R})$.

As it was shown above in this case the effect of noise generator on antenna with gain coefficient $\mathrm{G}_{\mathrm{R} 2}(\mathrm{R})$ woud be maximum and it effect on antenna with gain coefficient $\mathrm{G}_{\mathrm{R} 1}(\mathrm{R})$ would be minimum.

As regards the authenticity of limitation condition (8), imposed on gain coefficient of noise generators antenna the graphic interpretation of this condition shown in figure 8 make it possible to interprete condition (8) in the form of three strategies for organization of protection from noise effects:

- At the beginning of tracking cycle, when the distance between tracked object (for example, guided aviation bomb) and noise generator is maximum noise of 
maximum power should be generated, (curve 1 at the fig.8.)

- At the middle of moving objects tracking cycle the noise of maximum value should be generated (curve 2 at fig.8).

- On minimum possible distance between between object and noise generator, at the end of tracking cycle the noise of maximum value should be generated. (curve 3 at fig.2).

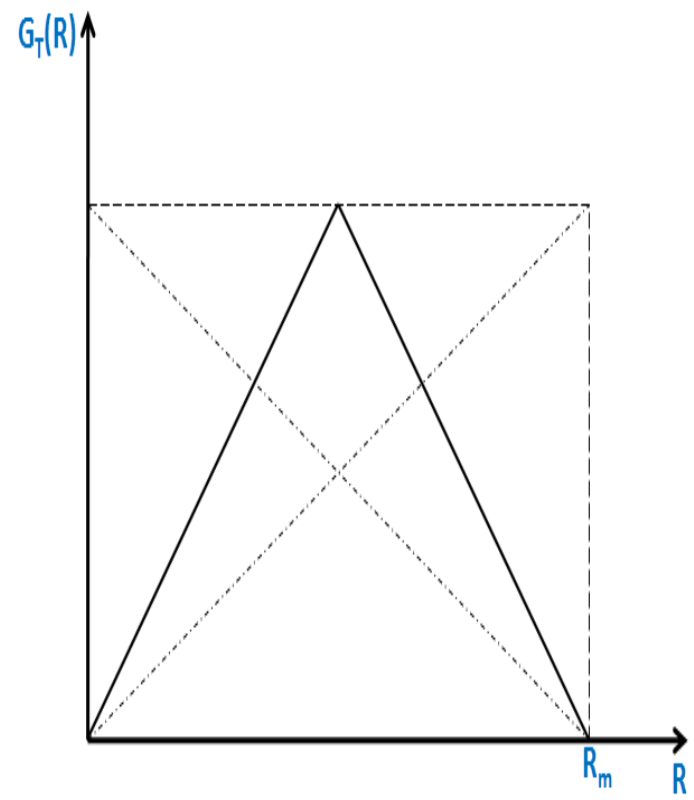

Fig.2. Possible curves of variation of $\mathrm{G}_{\mathrm{T}}(\mathrm{R})$ at the distance $0-R_{m}$, in line with accepted strategy for generation of noise signal.

\section{REFERENCES}

[1] The Friis Equation. RF \& Microwave Knowledge Center.

[2] L.A. Faria, C.A.M. Silvestre, M.A.F.Correia. GPS - dependent systems: Vulnerabilities to Electromagnetic Attacks. Journal of Aerospace Technology Management. V.8, №4, pp. 423 - 430, 2016.

[3] S. Lakshmi, N. Prasad. GPS Receiver Protection Requirement for Unmanned Aerial Vehicle. International Journal of Electrical, Electronics and Computer Engineering, 1(2), pp. 55-59, 2012

[4] G.T. Schmidt. INS/GPS Technology Trends. RTO-EN-SET-116, 2011. PP. 1-24. http://www.dtic.mil/dtic/tr/fulltext/u2/a581015.pdf

[5] GPS Carrier-to-Noise density. http://www.northwoodlabs.com/AN101.pdf.

[6] O.C.Dabak, F.Fradem, T.Sonmer, L.Alatan, S.SengerKoc, Havelsan A.S. Interference Supression in a GPS receiver with 4 element Array design and implementation of beamforming algorithms. https://www.semanticscholar.org/paper/Interference-suppression-in-aGPS-receiver-with-4-Dabak-Erdem/1d7978369587ecee9e0a53a70caa 2582e9a23942

[7] Sh.Sh. Jan,Ch.Ch. Sun. Signal existence verification (SEV) for GPS low received power signal detection using the time-frequency approach. Sensors 2010, 10, pp.4717-4738.

[8] William Bamford, Luke Winternitz, Curtis Hay. GPS World, Innovation. Autonomous navigation at high Earth orbits. https://ntrs.nasa.gov/search.jsp?R=20060012296

[9] J. 1. Ruiz.,Ch.H. Frey. Geosynchronous satellite use of GPS. ION GNSS 18th International Technical Meeting of the Satellite Division, 13-16 September, 2005, Long Beach, Ca. pp. 1227-1232.

[10] Andre Hauschild, MarkusMarkgraf, Oliver Montenbruck. The navigation and occultation experiment. GPS receiver performance on board a LEO satellite. Inside GNSS. July/August 2014. www.insidegnss.com. P.49-58 